\title{
Size quantization effects in optical and electrical properties of II-VI semiconductor films in nanocrystalline form
}

\author{
A K PAL \\ Department of Materials Science, Indian Association for the Cultivation of Science, Caicutta 700 032, India
}

\begin{abstract}
The electrical properties of CdTe and optical properties of $\mathrm{ZnS}$ in nanocrystalline thin film form are studied with a view to have a clearer understanding of the optical processes and the carrier transport mechanisms in nanocrystalline II-VI semiconductors, in general. Nanocrystalline ZnS and CdTe films were deposited by magnetron sputtering of respective targets in argon plasma. The optical absorption data of nanocrystalline $\mathrm{ZnS}$ films (thickness $10-40 \mathrm{~nm}$ ) could be explained by the combined effects of phonon and inhomogeneity broadening along with optical loss due to light scattering at the nanocrystallites. The conductivity of CdTe (grain size within $4-4.7 \mathrm{~nm}$ ) showed $\left(T_{0} / T\right)^{p}$. dependence with $p-0.5$ indicating the presence of a Coulomb gap near the Fermi level. The width of the Coulomb gap varied within 0.02-0.04 eV depending on the deposition condition. The existing theoretical models were used for estimating hopping energy $(0.02-0.04 \mathrm{eV})$ and hopping distance $(2 \cdot 8-5 \cdot 1 \mathrm{~nm})$ in nano CdTe films.
\end{abstract}

Keywords. Nanocrystalline films; optical and electrical properties.

\section{Introduction}

In recent years, there has been growing interest in the synthesis of II-VI semiconductors in nanocrystalline thin film form. Among different II-VI binary semiconducting materials, $\mathrm{ZnTe}, \mathrm{ZnSe}, \mathrm{ZnS}, \mathrm{CdSe}, \mathrm{CdS}$ and $\mathrm{CdTe}$ have received much attention due to their various device applications. Semiconductor nanostructures or quantum dots of these materials are very attractive since they exhibit interesting properties involving singularities in the density of states and improved linear and nonlinear optical responses (Yoffe 1993; Woggon and Gaponenko 1995).

Semiconductor nanocrystallites or zero dimensional quantum dots are significantly different from other low dimensional quantum structures as they consist of as few as $10^{3}-10^{6}$ atoms. A considerable fraction of these atoms reside on the surfaces of the devices or quantum dots. It is not very clear yet how the electronic states of these quantum dots are affected by the surrounding medium although the optical properties of the quantum dots show blue shift due to carrier confinement. The blue shift of $\mathrm{ZnTe}$ and $\mathrm{ZnSe}$ nanocrystalline films resulting from three-dimensional quantum confinement has recently been reported (Pal et al 1994; Banerjee et al 1997). In general, the blue shift in quantum dots increases with the decrease in the dimension of the crystallites whereas in a quantum well structure the quantum confinement becomes most effective when the well width becomes smaller than the de Broglie

*Author for correspondence wavelength of electron. The carrier confinement resulting from band structure modification produces interesting physical properties with potential application in optical devices such as lasers and photodetectors (Tsang 1987; Schmitt-Rink et al 1989). The quantum confinement stabilizes the excitonic effect and photoluminescence due to the excitonic recombination may be expected at a higher temperature.

Although considerable progress has been made in the theoretical understanding of the optical processes in nanocrystalline semiconductors, the electrical properties of these materials have not received the attention they deserve. Recently, extensive studies on optical and electrical properties of $\mathrm{ZnTe}$ (Pal et al 1994; Maity et al 1995), ZnSe (Banerjee et al 1997; Mandal et al 1998), CdS (Gupta et al 1995), ZnS, and CdTe in nanocrystalline thin film were carried out by us and other workers. In this communication, we present the electrical and optical properties of II-VI semiconductors in nanocrystalline thin film form with a view to have a clearer understanding of the optical processes and the carrier transport mechanism and their dependence on the sizes of the nanocrystallites. We will discuss the optical properties by taking $\mathrm{ZnS}$ as a representative material and $\mathrm{CdTe}$ will be a representative material while discussing electron transport processes.

\section{Experimental}

Nanocrystalline $\mathrm{ZnS}$ and CdTe were deposited in thin film form at low substrate temperatures by d.c. magnetron sputtering of appropriate targets (of $99.999 \%$ purity) in 
argon plasma at a gas pressure of $\sim 20-40 \mathrm{~Pa}$. The target to substrate distance was $\sim 2.5 \mathrm{~cm}$. The deposition was performed at $\sim 1.5 \mathrm{KV}$ and $\sim 100 \mathrm{~mA}$. Before starting the deposition, the target was presputtered for appropriate duration with a shutter covering the substrate. In high pressure sputtering, the particles ejected from the target undergo many collisions with the argon gas atoms due to the high chamber pressure and subsequently they nucleate and grow by rapid condensation on the substrate to form ultrafine particles. The substrate holder was a thick copper plate $(10 \mathrm{~mm}$ thick) which served as a cold finger (cooled by liquid nitrogen). Intermediate temperatures could be attained by heating the copper substrate holder by appropriate heating elements passed through holes laterally drilled through the copper plate. The temperature of the substrate was measured and controlled $\left( \pm 1^{\circ}\right)$ by a copper-constantan thermocouple acting as a probe to an electronic on/off temperature controller.

It was observed that when the deposition was carried out at a stretch for a longer time ( $>2 \mathrm{~min}$ ), the substrate temperature increased due to the impact of the particles ejected from the target. This resulted in an increase of grain size through the increased adatom mobilities culminating in coalescence of the crystallites. Thus, films with discrete particles in nanometric scale could not be obtained by this process for higher film thicknesses and as such the thicknesses of the films were always limited to $\ll 10 \mathrm{~nm}$. In order to overcome this, the deposition was carried out intermittently at regular interval to allow the oncoming adatoms release their thermal energy before a second deposition was made. An electronically controlled shutter, used for this purpose, was logically connected to the output of a thermocouple (used for measuring and controlling the substrate temperature during deposition). This sequential sputtering technique was very useful in obtaining nanocrystalline film with controlled grain size throughout the thickness.

Three sets of CdTe films were deposited with substrate temperatures $\left(T_{s}\right) 253,263$ and $273 \mathrm{~K}$. For each of these $T_{\mathrm{s}}$ values films were deposited for different deposition times $(5,10$ and $15 \mathrm{~min}$ ) producing films of thickness within $10-40 \mathrm{~nm}$. However, the $\mathrm{ZnS}$ films were deposited only for $3 \mathrm{~min}$ duration.

Transmission electron microscopy (Hitachi H-600) was used to obtain microstructural information and average grain size was evaluated from it. The grain sizes were also determined from the theoretical curve fitting of the photoluminescence (PL) and optical absorption spectra. PL measurements were carried out at $79 \mathrm{~K}$ using $300 \mathrm{~mW}$ $\mathrm{Xe}$ arc lamp as the excitation source, 3/4 meter monochromator and Hamamatsu photomultiplier as detector. The CdTe samples were excited with $600 \mathrm{~nm}$ and $700 \mathrm{~nm}$ radiation while $\mathrm{ZnS}$ samples were excited with $300 \mathrm{~nm}$ radiation. The electrical conductivities $(\sigma)$ of the films were measured in the temperature range $188-298 \mathrm{~K}$ by the four-probe technique with a constant current source (Keithley 225) and an electrometer (Keithley 6517A). Predeposited Ni contacts (deposited by evaporation) were used for the conductivity measurement of CdTe films. The substrate was quartz for electrical/optical measurement and $\mathrm{NaCl}$ was used for the TEM studies.

\section{Results and discussion}

\subsection{Nanocrystalline $\mathrm{ZnS}$ film}

3.1a Determination of optical band gap: Transmission electron diffraction studies of the nanocrystalline $\mathrm{ZnS}$ films indicated zinc blende structure (figure 1). The absorption coefficients $(\alpha)$ of the $\mathrm{ZnS}$ films were evaluated by measuring transmittance and reflectance (Bhattacharyya et al 1992) by a UV-VIS-NIR spectrophotometer (Hitachi U-3410). The band gaps $\left(E_{\mathrm{g}}\right)$ were determined by extrapolating $(\alpha h v)^{2}$ versus photon energy $(h v)$ plots (not shown here). The absorption trace for a representative film of nanocrystalline $\mathrm{ZnS}$ deposited at a substrate temperature of $\sim 213 \mathrm{~K}$ with $30 \mathrm{~min}$ of deposition time is shown in figure 2 . It may be noted that the variation of the absorption $(\alpha)$ with the wavelength $(\lambda)$ of the incident radiation were nearly the same for all the films studied here. It was noticed that the fall of the absorption tail was sharper for films deposited for higher deposition time than those deposited for lower duration (i.e. for thinner films).

Figure 3 shows the variation of band gaps of several nanocrystalline $\mathrm{ZnS}$ films deposited at different substrate temperature and deposition time. The band gap values were always found to be higher compared to the bulk value. It may be noted that the films deposited at $213 \mathrm{~K}$ showed the maximum shift $\left(\Delta E_{\mathrm{g}}=E_{\mathrm{g}}-E_{\mathrm{g}(\mathrm{bulk})}\right)$ of band

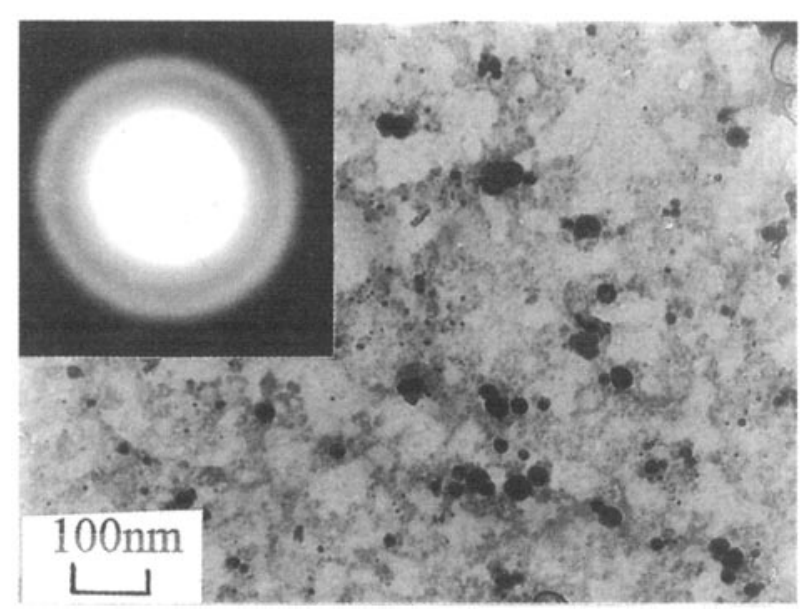

Figure 1. TEM micrograph and the corresponding diffraction pattern of a representative nanocrystalline $\mathrm{ZnS}$ film deposited at $T_{\mathrm{s}}=233 \mathrm{~K}$ and $40 \mathrm{~Pa}$. 
gap from its bulk value $(3.6 \mathrm{eV})$. The amount of shift in the band gap $\left(\Delta E_{\mathrm{g}}\right)$ decreased substantially as the deposition temperature was increased. This is basically due to the fact that the films deposited at lower substrate temperature had smaller nanocrystallite sizes which was reflected in larger blue shift. Presence of smaller crystallite sizes would indicate the presence of an increasing amount of amorphous phase mixed with the nanocrystallites. This has been reflected in the gradual departure of the sharpness of the fall of the trace of transmittance (Tr) versus wavelength $(\lambda)$ for films deposited at lower substrate temperatures. The average particle size, $(\bar{R})$, was measured from TEM studies and also from the curve fitting of the absorption data (discussed in later section of paper). It was observed that for a constant gas pressure in the chamber, the average particle size, $(\bar{R})$, increased (figure 4 ) with the increasing substrate temperature $\left(T_{\mathrm{s}}\right)$ up to $\sim 300 \mathrm{~K}$ after which a sharp increase in the grain size was observed with increasing $T_{\mathrm{s}}$. No agglomeration was observed till $T_{s}-300 \mathrm{~K}$ as the gas pressure probably did not cross the threshold limit for agglomeration. The crystallite size did not vary significantly as the deposition time was increased. We have also measured the band gaps of $\mathrm{ZnS}$ films deposited at higher temperature $(>320 \mathrm{~K})$ which indicated that the films became polycrystalline with band gaps nearly equal to the bulk value.

The increase of the fundamental band gap of the nanostructured materials due to the quantum size effect (QSE) is well established. For the sake of comparison, we estimated the band gap of these nanocrystalline films from Kayanuma (1988):

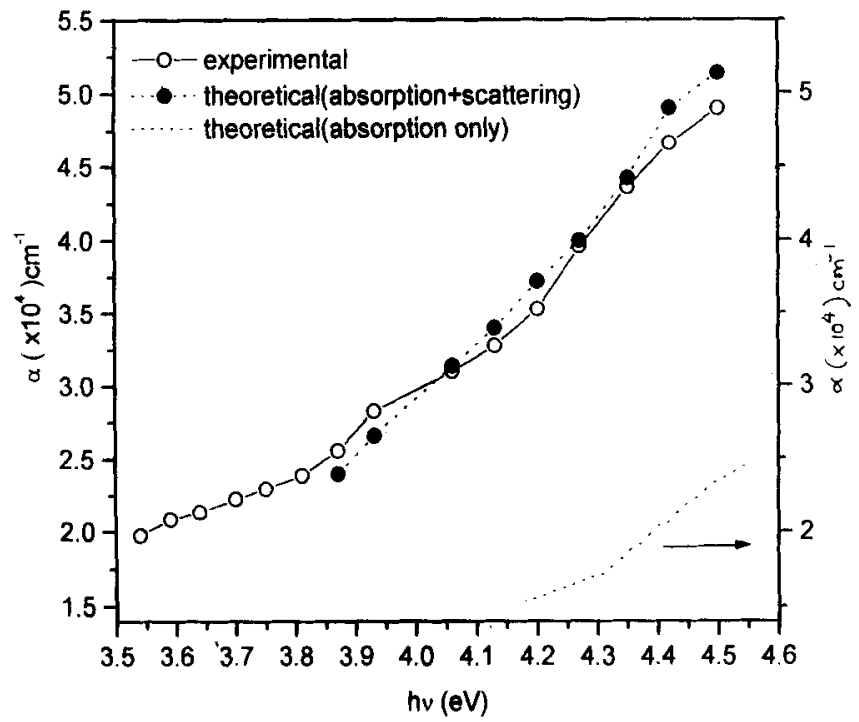

Figure 2. Variation of absorption coefficient $(\alpha)$ with wavelength $(\lambda)$ for a representative $\mathrm{ZnS}$ film deposited at a substrate temperature $\left(T_{\mathrm{s}}\right)$ of $\sim 213 \mathrm{~K}$ with $30 \mathrm{~min}$ duration of deposition.

$$
E_{\mathrm{g}}=E_{\mathrm{g}}^{0}+\frac{\hbar^{2} \pi^{2}}{2 \mu R^{2}}-1.786 \frac{e^{2}}{4 \pi \varepsilon_{0} R}-0.248 E_{\mathrm{RY}}^{*}
$$

for the strong confinement limit (i.e. $R \ll a_{\mathrm{B}}$ ) of QSE in nano material. For weak confinement limit (i.e. $R \gg a_{\mathrm{B}}$ ) we have:

$$
E_{\mathrm{g}}=E_{\mathrm{g}}^{0}+\frac{\hbar^{2} \pi^{2}}{2 M R^{2}}-E_{\mathrm{RY}}^{*}
$$

where $M=m_{\mathrm{e}}+m_{\mathrm{h}}, m_{\mathrm{e}}$ and $m_{\mathrm{h}}$ being the electron and hole effective masses, respectively. In (1), $R$ is the radius of the nanocrystallites, $E_{\mathrm{RY}}\left(=e^{2} / 4 \pi \varepsilon_{0} a_{\mathrm{B}}\right)$ the effective Rydberg energy for the bulk exciton with $a_{\mathrm{B}}$ as the corresponding Bohr radius $\left(=4 \pi \varepsilon_{0} \hbar^{2} / \mu e^{2}\right), \mu=\left[m_{\mathrm{e}} m_{\mathrm{h}} /\right.$ $\left.\left(m_{\mathrm{e}}+m_{\mathrm{h}}\right)\right]$ being the reduced mass. $E_{\mathrm{g}}^{0}$ and $\varepsilon_{0}$ are the band gap and low frequency dielectric constant, respectively of the bulk material. By utilizing the average particle size $(\bar{R})$, values as obtained from TEM measurements, the $E_{\mathrm{g}}$ values of different films were estimated from the above equation which were found to agree well with those obtained directly from the absorption spectra.

3.1b Absorption behaviour: As the films consist of large number of nanocrystallites having different sizes and shapes, the absorption peak corresponding to level to level interband transitions will be less sharp (figure 2) due to the enhanced phonon broadening and inhomogeneity broadening effects (Schmitt-Rink et al 1987; Pal et al 1994).

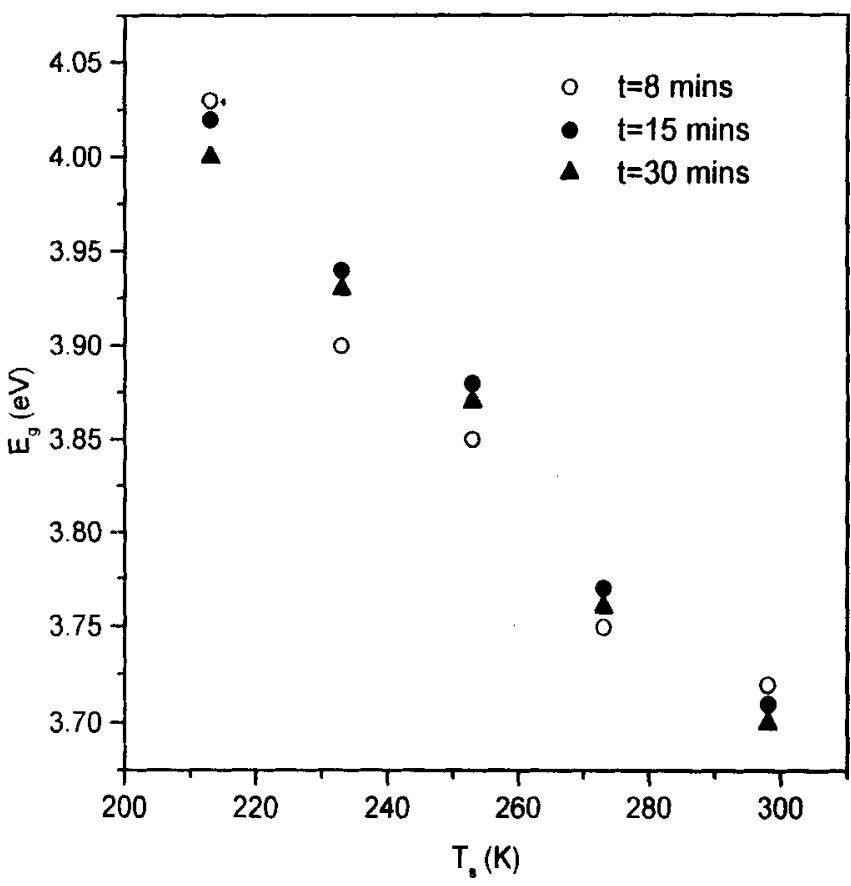

Figure 3. Variation of band gap $\left(E_{\mathrm{g}}\right)$ for $\mathrm{ZnS}$ films with substrate temperature $\left(T_{s}\right)$ when deposited at various deposition times. 
The origin of the broadening due to inhomogeneity is generally ascribed to the fluctuation in the shape and sizes of the grains in the film. The deviation from the perfectly spherical shape of the particles would likely modify the selection rule and the transitions forbidden for the spherical form might appear in real situation. Moreover, the size distribution of the nanocrystallites would culminate in the fluctuation in the energy subbands as inferred from (1) and (2). Thus, the superposition of all the discrete absorptions due to these combined effects will result in broadening of the experimentally observed spectrum. It may be mentioned here that in this treatment, we have not taken into account the effect of the fluctuation in the shape of the crystallites on the absorption spectrum due to the inherent complexity of the problem.

The broadening due to phonon would also exist besides the above inhomogeneous broadening. This intrinsic broadening would have a finite value at room temperature. Klein et al (1990) considered this energy broadening due to phonon interaction in nanocrystalline systems and assuming the nanocrystallites to be spherical in shape having Gaussian size distribution, the total absorption was expressed as (Allegre et al 1994; Pal et al 1994):

$$
\alpha=\int_{R_{\min }}^{R_{\max }} P(R) \alpha(R) \mathrm{d} R
$$

where $P(R)$ is the probability of size distribution given by:

$$
P(R)=\left(R-R_{\min }\right)\left(R_{\max }-R\right) \exp \left(-\frac{R^{2}}{\delta^{2}}\right),
$$

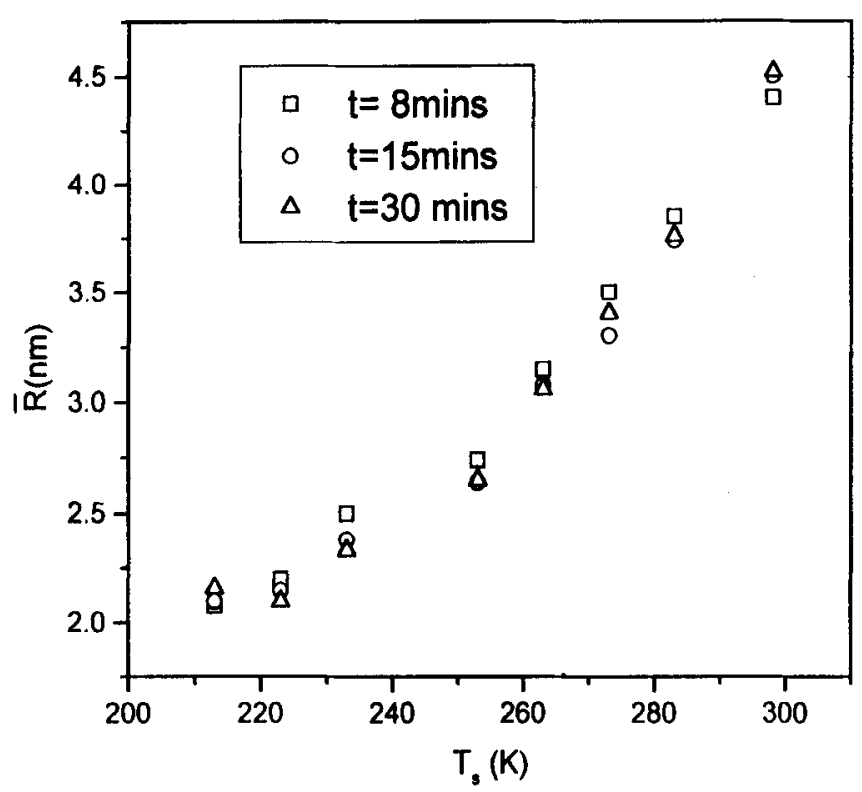

Figure 4. Variation of crystallite sizc $(\bar{R})$ with substrate temperature $\left(T_{s}\right)$ for films deposited at various deposition times. where $\delta=0.76, R=\left(R_{\max }+R_{\min }\right) / 2 . \alpha(R)$ is the absorption coefficient due to a nanocrystallite of radius $R$ and can be written as (Schmitt-Rink et al 1987; Pal et al 1994):

$$
\alpha(R)=\frac{\omega}{n_{0} c \varepsilon_{0}}\left(C_{0} / V\right) \frac{\hbar \Gamma}{\left(E_{\mathrm{g}}-\hbar \omega\right)^{2}+(\hbar \Gamma)^{2}},
$$

with,

$$
C_{0}=\frac{2 \pi e^{2} \hbar^{2}}{m_{\mathrm{e}}^{2}(\hbar \omega)^{2}}\left|p_{\mathrm{cv}}\right|^{2},
$$

where $\hbar \omega$ is the photon energy, $n_{0}$ the refractive index, $c$ the velocity of light, $V$ the volume of the spherical nanocrystallite and $p_{c v}$, the bulk optical matrix element, and is given by (Yoffe 1993):

$$
\left|p_{\mathrm{cv}}\right|^{2}=\left(m_{\mathrm{e}} / 3\right) \frac{E_{\mathrm{g}}\left(E_{\mathrm{g}}+\Delta\right)}{\left(E_{\mathrm{g}}+2 \Delta / 3\right)},
$$

$\Delta$ being the spin-orbit splitting factor.

3.1c Optical loss due to scattering: It is apparent from figure 2 that the optical absorption solely cannot explain the shape and magnitude of the experimental absorbance spectra at room temperature. It is true that a large amount of incident light would be scattered by the ultra small crystallites. In this case, the total intensity $\left(I_{\mathrm{SC}}\right)$ of the scattered light per unit volume for normal incidence may be given by using the Mie scattering theory (Born and Wolf 1959). In the limit where the crystallite dimensions are much less than the optical wavelength $(\lambda)$ :

$$
l_{\mathrm{sC}}=\int P(R) I_{\mathrm{sc}}(R) \mathrm{d} R,
$$

with

$$
I_{\mathrm{sc}}(R)=(2 / V)\left[(2 \pi / \lambda) R^{3} \frac{\left(n_{0}^{2}-1\right)}{\left(n_{0}^{2}+2\right)}\right]^{2} .
$$

It may be noted that we did not consider the absorption due to surface traps and other impurities in our calculation as pointed out by Sekikawa et al (1992). The theoretically simulated absorbance spectra for $\mathrm{ZnS}$ nanostructured films were obtained by using (3) and (8) and are shown in figure 2 . The amount of the optical loss due to scattering effect is easily estimated from the comparison between the theoretically simulated absorbance and absorption curves. The values of different parameters utilized to compute the above simulated absorbance spectra are as follows: $E_{\mathrm{g}}=3.68 \mathrm{eV} ; \quad m_{\mathrm{e}}=0.27 m_{0} ; \quad m_{\mathrm{h}}=0.58 m_{0}$; $\varepsilon_{0}=8.1 ; \varepsilon_{\alpha}=5.13 ; \Delta=0.067 ; \omega_{\mathrm{LO}}=10.44 \mathrm{THz}$ for $\mathrm{ZnS}$ where, $m_{0}$ is the free electron mass. It can be observed that the simulated spectra fitted well with $\bar{R}$ value of $3 \mathrm{~nm}$ (with a mean fluctuation of $\pm 0.5 \mathrm{~nm}$ ) for $\mathrm{ZnS}$. This agreed well with the nanocrystallite sizes as determined from the TEM studies and the shift in band gap. 
3.1d Photoluminescence studies: Photoluminescence (PL) spectra of the $\mathrm{ZnS}$ nanocrystalline films were recorded at $100 \mathrm{~K}$ with an exciting wavelength of $300 \mathrm{~nm}$. The PL spectra of the films had a peak in the range $350-400 \mathrm{~nm}$. It can be seen (figure 5) that the line shapes of the PL spectra are asymmetric and broad. The film deposited at lower substrate temperature showed broader PL peak. Shift of these PL peaks to higher energies for films deposited at lower substrate temperature (i.e. with lower crystallite size) was visible for all the films irrespective of their thicknesses (e.g. deposition time).

The appearance of the PL peaks at energies substantially lower than the band gap suggests that transitions from energy states in the band gap are being favoured for the luminescence process in these nanocrystalline $\mathrm{ZnS}$ films. We would like to ascribe this observation to the presence of surface states of $\mathrm{ZnS}$ nanocrystallites constituting the film. This observation agrees well with that by Chen et al (1997). The assignment of the surface states for the observed PL peak position at energies lower than band gap energy was also supported by the fluorescence and thermoluminescence measurements made by them. The density of surface states of nanocrystalline films would increase with the decrease in crystallite size constituting the film due to the increase in surface to volume ratio in films having smaller crystallites. This would reduce the probability of excitonic emission via nonradiative surface recombinations (Chen et al 1997; Sekikawa et al 1992). The band edge or excitonic emission will thus be effectively overlapped with the absorption of the surface states and as such would show photoluminescence at energies less than the band gap. The position of the PL peak would depend on the size of the nanocrystallite i.e. the substrate temperature during deposition. It was observed that the films deposited at lower temperature had lower crystallite size and the PL peak was shifted to higher energies. It is also interesting to note that thinner films deposited at a particular substrate temperature indicated peak position (figure 5a) at lower energies than that for a thicker film (figure $5 b$ ) deposited at the same substrate temperature. This is commensurate with the fact that the surface to volume ratio will be higher for thinner films than that of thicker films. Thus the observed PL spectra supports the so-called trapped luminescence arising from the surface states. Similar crystallite size dependence of the shift of surface emission has also been reported by Chestnoy et al (1986).

\subsection{Nanocrystalline CdTe film}

Nanocrystalline thin films, in general, consist of grains and grain boundaries in the nanometer range and they are characterized by the presence of large surface to volume ratios of the crystallites constituting the film.
This gives rise to properties different from those in the bulk in which there are large grains with a long range order. Grain boundaries, in general, play a dominant role in the nanocrystalline materials because a large fraction of the atoms in the nanocrystalline materials are located only within a few atomic distances of the grain boundaries. Understanding the origin of the unique properties of nanocrystalline materials requires complete characterization of the sample by utilizing the existing theoretical models.

3.2a Structural studies: Nanocrystalline CdTe films deposited at substrate temperatures $\left(T_{s}\right) \sim 253,263$ and $273 \mathrm{~K}$, were examined under transmission electron microscope (TEM). Diffused rings could be observed in the TEM diffraction patterns for all the films deposited at $T_{\mathrm{s}} \leq 273 \mathrm{~K}$. Figures $6 \mathrm{a}$ and $\mathrm{b}$ show the micrograph and the corresponding diffraction pattern of a representative film deposited at $273 \mathrm{~K}$ for $5 \mathrm{~min}$. It may be noted that distinct rings begin to appear for films deposited at $T_{\mathrm{s}}>273 \mathrm{~K}$. Thus, we have limited the highest temperature of deposition for the nanocrystalline CdTe

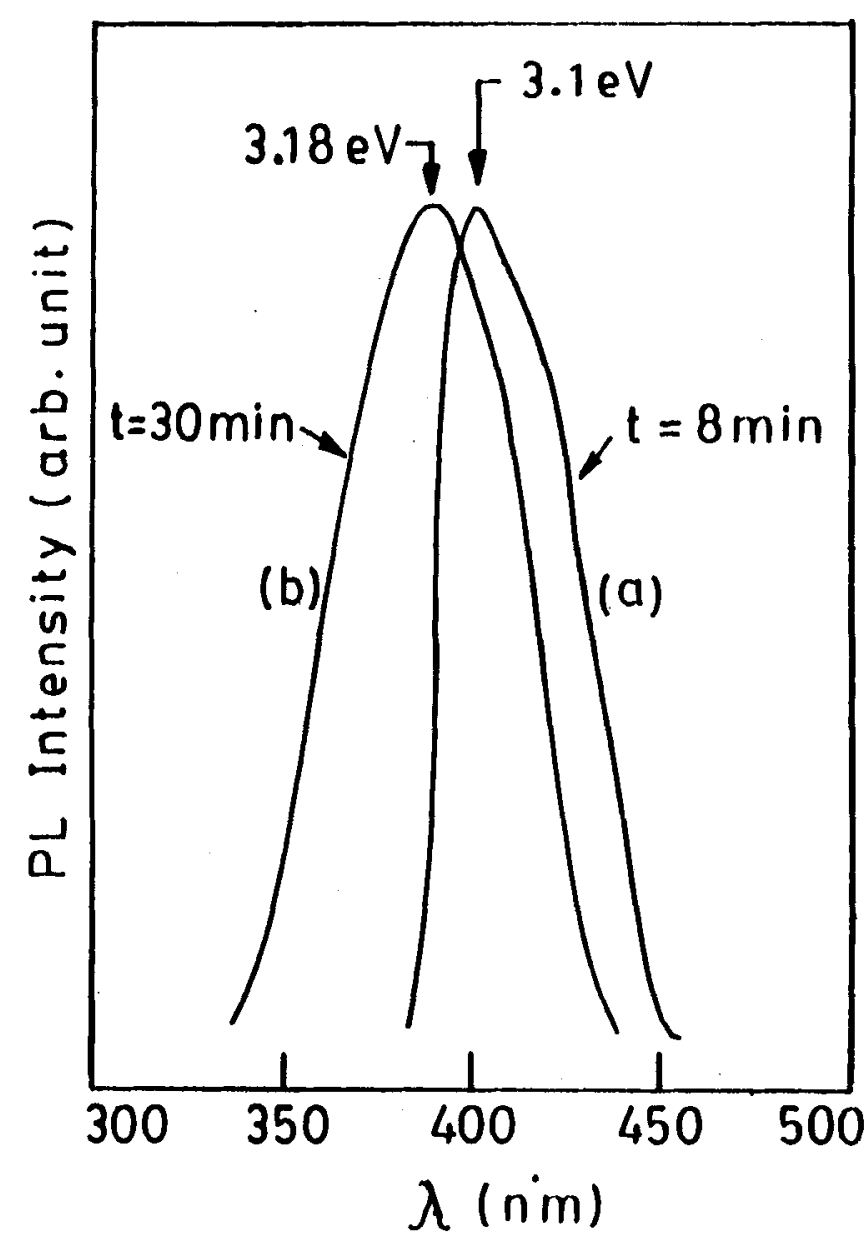

Figure 5. Photoluminescence spectra for two representative $\mathrm{ZnS}$ films deposited at $233 \mathrm{~K}$. 
films to $\leq 273 \mathrm{~K}$. The crystallite sizes were found to vary with $T_{s}$. It may be noted from figure $7 \mathrm{a}$ that the average crystallite sizes $(d)$ decreased with the decrease of deposition temperature from $273-253 \mathrm{~K}$ for different thicknesses of the films (corresponding to deposition times of 5,10 and $15 \mathrm{~min}$ ). It may also be noted (figure 7a) that the grain size is slightly less for sequentially sputter-deposited films deposited for higher duration than that for films sputtered continuously at same $T_{\mathrm{s}}$ even for lower duration. This indicates a definite departure from the conventional grain growth. We have observed that grain size could be kept substantially low by using the sequential deposition technique instead of depositing the film at a stretch for the same time when increase of substrate temperature resulted in an increase of grain size. The beauty of the sequential deposition technique is that it is now possible to deposit nanocrystalline CdTe films (for $T_{\mathrm{s}} \leq 273 \mathrm{~K}$ ) with nearly uniform crystallite size having appreciable thickness.
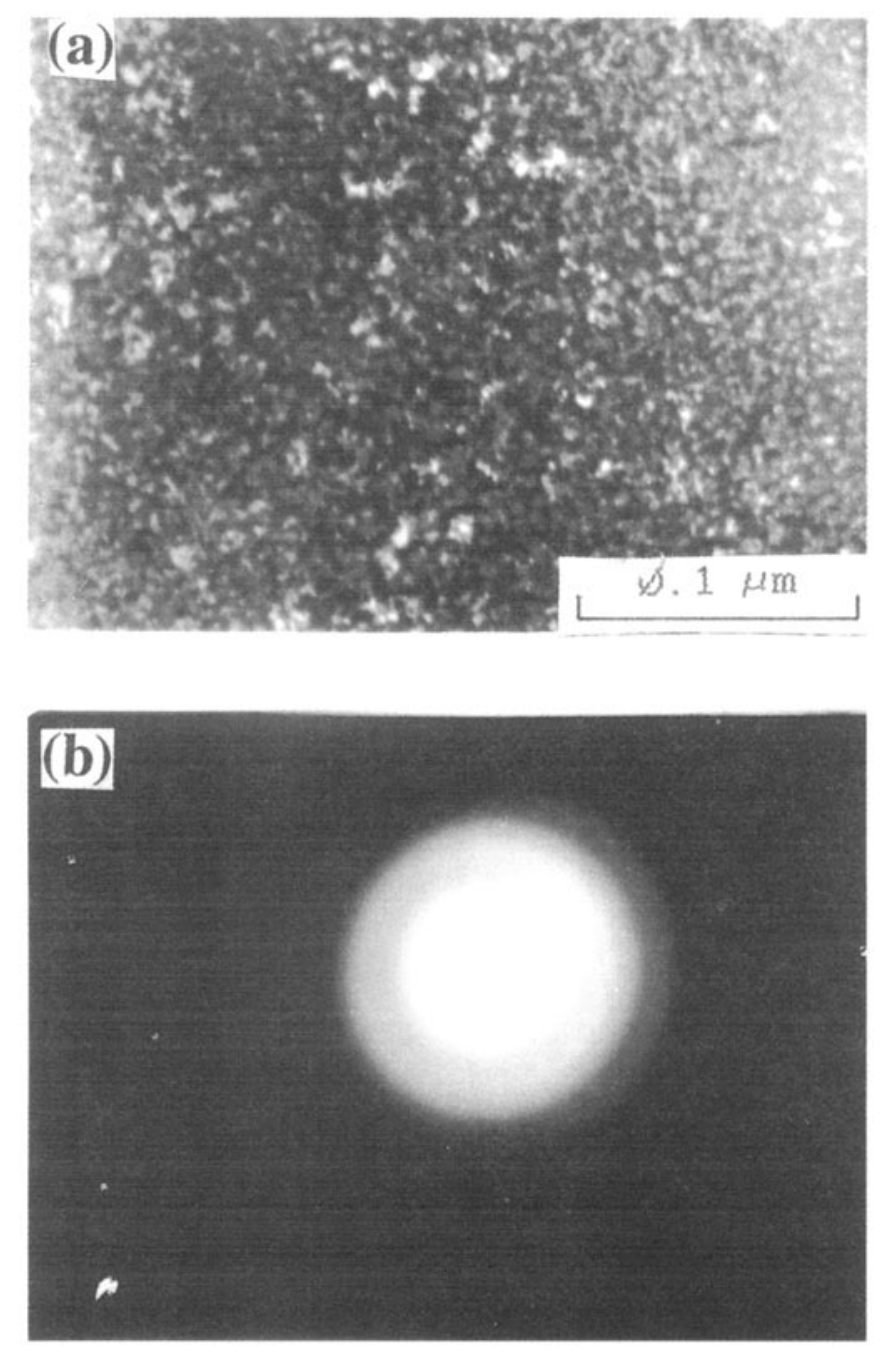

Figure 6. (a) Transmission electron micrograph and (b) diffraction pattern of a representative CdTe film deposited at a substrate temperature $\left(T_{\mathrm{s}}\right) \sim 273 \mathrm{~K}$. 3.2b Optical measurements: Transmittance $\left(T_{\mathrm{r}}\right)$ versus wavelength $(\lambda)$ data were recorded by a spectrophotometer (Hitachi U-3410) and absorption coefficients $(\alpha)$ were evaluated from these by standard procedure. The optical band gaps $\left(E_{\mathrm{g}}\right)$ of the CdTe films with different grain sizes are determined from $(\alpha h v)^{2}$ vs $h v$ plots. Figure $7 \mathrm{~b}$ shows that $E_{\mathrm{g}}$ increased from $2 \cdot 18-2.45 \mathrm{eV}$ with decrease of grain diameter from 4.71 to $4.05 \mathrm{~nm}$ corresponding to the deposition time of $5 \mathrm{~min}$. The variation of $E_{\mathrm{g}}$ for deposition times of 10 and $15 \mathrm{~min}$ are also shown in figure $7 \mathrm{~b}$. It should be noted that the $E_{\mathrm{g}}$ values in our nanocrystalline CdTe films were higher than the band gap of bulk CdTe $(1.45 \mathrm{eV})$. With the increase in deposition time, the band gaps slightly increased (figure 7b) due to the observed decrease of grain size (figure 7a). The increase of band gap with decreasing grain size indicated blue shift due to quantum confinement in the CdTe nanocrystallites having radius $R<a_{\mathrm{B}}$, where $a_{\mathrm{B}}$ is the Bohr radius of bulk exciton. It may be noted that the value of $a_{\mathrm{B}}$ for bulk CdTe $(\sim 7 \mathrm{~nm})$ is much higher than the average radius $(2.0-2.4 \mathrm{~nm})$ of the CdTe nanocrystallites in our films. It may also be observed that when the sequential deposition time was increased, the band gaps did not decrease which was contrary to the films deposited at a stretch for the same total time. This showed that the sequential deposition technique was very effective in maintaining constant grain size throughout the thickness of the film.

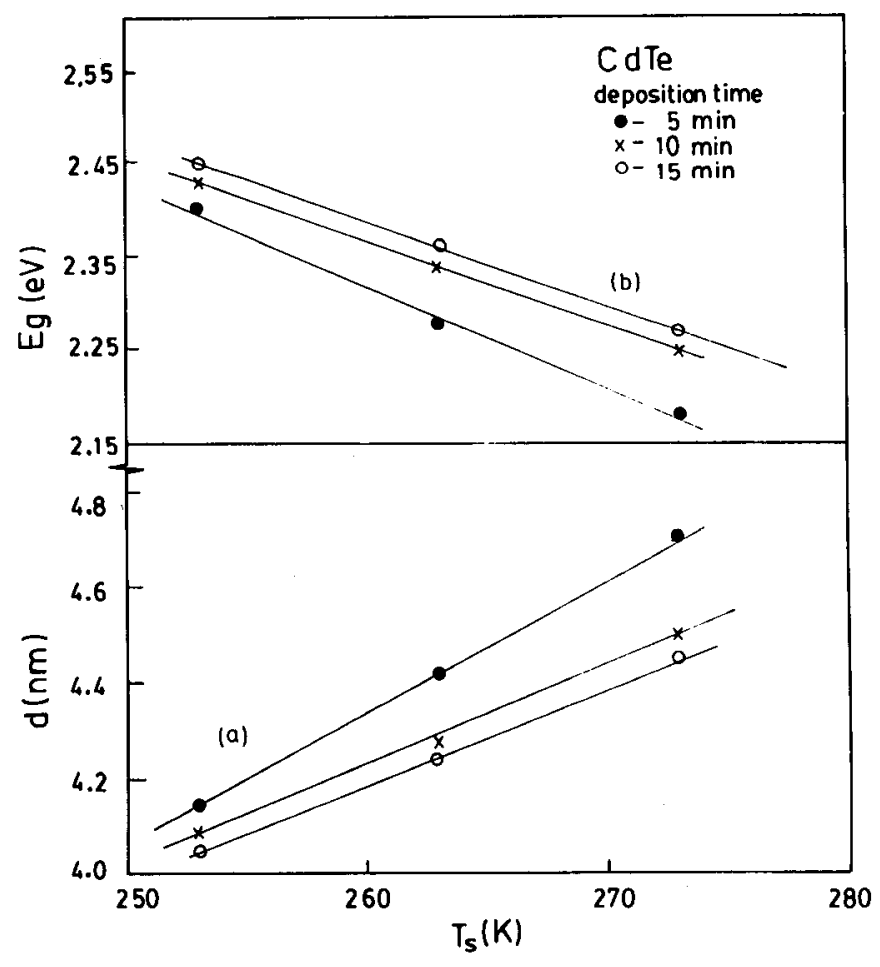

Figure 7. Variation of (a) grain size and (b) optical band gap $\left(e_{g}\right)$ with deposition temperature $\left(T_{s}\right)$ of nanocrystalline CdTe films corresponding to deposition times of 5,10 and $15 \mathrm{~min}$. 
3.2c Electrical conductivity studies: The variation of electrical conductivity $(\sigma)$ of nanocrystalline CdTe films with temperature indicated (figure 8) typical Arrhenius type of activation:

$$
\sigma=\sigma_{0} \exp \left\{-E_{\sigma} / k T\right\},
$$

with the activation energy $\left(E_{o}\right)$ varying from $55 \mathrm{meV}$ (at higher temperature) to $13 \mathrm{meV}$ (at low temperature). Very low value of $E_{\sigma}$, specially at lower temperature, obtained here indicate that hopping of the carriers between the localized states within or outside the Coulomb gap may be the dominant transport mechanism in these films. The validity of the Efros-Shklovskii and Mott models (Efros and Shklovskii 1984; Mott 1968) for hopping transport in these highly resistive films have been examined in the following section.

3.2d Conductivity of the disordered system: For a disordered system, the conductivity $(\sigma)$ may be expressed as

$$
\sigma=\sigma_{0} \exp \left[-\left(T_{0} / T\right)^{p}\right]
$$

where the pre-exponential factor $\sigma_{0}$ may be either independent of $T$ or a slowly varying function of $T$, while $T_{0}$ is a constant of the material. The value of $p$ depends on the grain size and the temperature range of measurement. It may be 0.25 for Mott's hopping and 0.5 or 1 for Efros hopping depending on the variation of the density of states with energy within the Coulomb gap. As a consequence of the long range Coulomb interaction between the localized state electrons, the density of states (DOS) would tend to zero near the Fermi energy $\left(E_{\mathrm{F}}\right)$ resulting in a soft energy gap corresponding to the

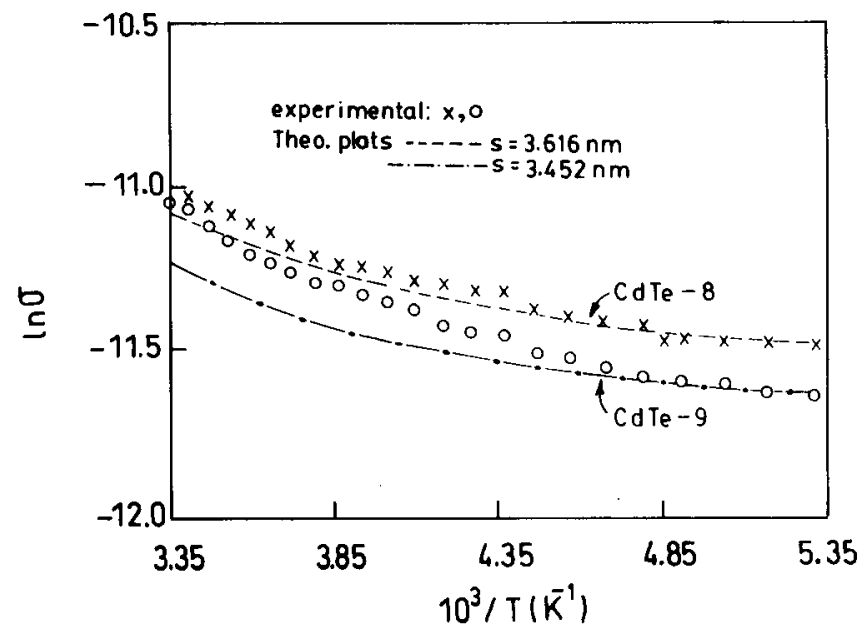

Figure 8. Experimental variation of electrical conductivity $(\sigma)$ of nano CdTe films with reciprocal temperature, along with the theoretical plots ( $s$ being the average distance between two consecutive grains). parabolic DOS. The idea of the Coulomb gap was first introduced by Efros and Shklovskii in 1975 (referred hereinafter as ES model) and the corresponding conductivity could be expressed as:

$$
\sigma=\sigma_{0} \exp \left[-\left(T_{0} / T\right)^{1 / 2}\right],
$$

where $T_{0}=e^{2} / \varepsilon_{\mathrm{r}} a, a^{-1}$ and $\varepsilon_{\mathrm{r}}$ being the electron localization range and the dielectric constant of the material, respectively. It is known that the effect of the Coulomb gap is not so prominent when the temperature of measurement $(T)$ of the sample is such that $k T$ becomes of the order of or greater than the Coulomb gap. Hopping of the carriers in the localized states outside the Coulomb gap then becomes predominant and the conductivity obeys the Mott's (1968) relation:

$$
\sigma=\sigma_{0} \exp \left[-\left(T_{0} / T\right)^{1 /(1+x)}\right],
$$

where $x$ is the dimensionality of the system. According to Mott (1968), the DOS around $E_{\mathrm{F}}$ should vary slowly or should be nearly constant, rather than being zero. For Mott's hopping in three-dimensional system, $x=3$ and $T_{0}=\beta \alpha_{\mu}^{3} / k g_{0}$, where $\alpha_{\mathrm{m}}^{-1}$ is the decay length of the wave function, $g_{0}$ the Mott DOS at the Fermi level and $\beta$ a constant. The value of $T_{0}$ depends on the defect density or $g_{0}$ value in the material. We have determined $g_{0}$ in our CdTe films deposited with different grain sizes and thicknesses.

3.2e Determination of the exponent $p$ : The exponent $p$ was determined from the temperature dependence of the conductivity $(\sigma)$ by using the relation (Demichelis et al 1993):

$$
\ln \sigma(T)=\ln \sigma_{0}-\left(T_{0} / T\right)^{p}
$$

If we write (Zabrodski 1984),

$$
W(T)=\frac{\mathrm{d}[\ln \sigma(T)]}{\mathrm{d}[\ln (T)]},
$$

we can determine $p$ from the slope of the plot of $\ln [W(T)]$ vs $\ln (T)$ which should be a straight line. The above plots for three representative films deposited at $T \sim 263 \mathrm{~K}$ with deposition times of 5,10 and $15 \mathrm{~min}$ are shown in figure 9 . It may be observed that the average value of $p$ is within $0.5-0.6$ which is an indication of the variable range hopping occurring within the Coulomb gap (Efros-Shklovskii hopping). In this variable range hopping (VRH) process, the electrons jump from one localized state to the other in which there is an overlap of the wave functions (Graham et al 1992). The difference in the eigen energies of the two localized states involved in the transition is compensated by the absorption or emission of phonons. 


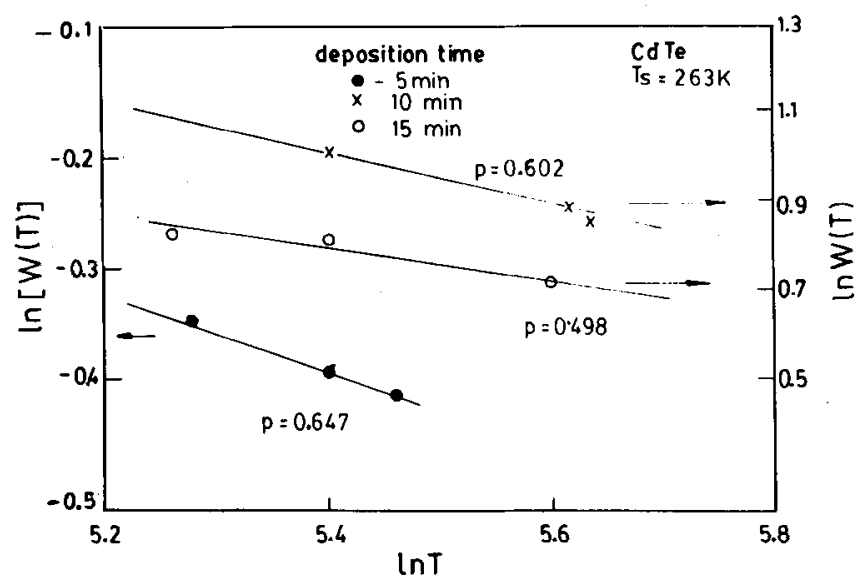

Figure 9. Plot of $\ln [W(T)]$ vs $\ln (T)$ for three representative CdTe films deposited at $T_{\mathrm{s}} \sim 263 \mathrm{~K}$ with deposition times of 5,10 and $15 \mathrm{~min}$.

3.2f Hopping parameters from ES model: We have used the ES model to determine the hopping parameters in the nanocrystalline CdTe films and investigated the justification of these parameters. Figure 10 shows the $\ln \left(\sigma_{0} / \sigma\right)$ vs $1 / T^{0.5}$ plots for five representative films. $T_{0}$ values were determined from the slope of the above plots by least square fit method and are shown in table 1 . Using these $T_{0}$ values, we calculated several other parameters e.g. hopping energy, hopping distance etc. The temperature dependent optimum hopping distance $\left(R_{\mathrm{opt}}\right)$ is given by:

$$
R_{\mathrm{opt}}=0.25 \alpha_{\mathrm{m}}^{-1}\left(T_{0} / T\right)^{1 / 2}
$$

Here $\alpha_{\mathrm{m}}$ is the tunneling exponent which may be obtained from:

$$
\alpha_{\mathrm{m}}=k T_{0}\left[\left(\pi g_{2}\right)^{1 / 3}\right] / 10 \cdot 5 .
$$

The value of $g_{2}$ in (8) is given by:

$$
g_{2}=3^{8} \pi^{2} \varepsilon_{\mathrm{r}}^{3} \varepsilon_{0}^{3} / 2^{5} e^{6}
$$

Here, $\varepsilon_{\mathrm{r}}$ is the dielectric constant of the material and $\varepsilon_{0}$ the free space permittivity and $e$ the electronic charge. For bulk CdTe, $\varepsilon_{\mathrm{r}}=10 \cdot 9$. Considering the parabolic variation of the density of localized states within the Coulomb gap, $g(E)$, may be expressed as:

$$
g(E)=g_{2}\left(E-E_{\mathrm{F}}\right)^{2}
$$

The average hopping energy $\left(W_{\text {opt }}\right)$ may be estimated from:

$$
W_{\mathrm{opt}}=0.5 \mathrm{k}\left(T_{0} T\right)^{1 / 2}
$$

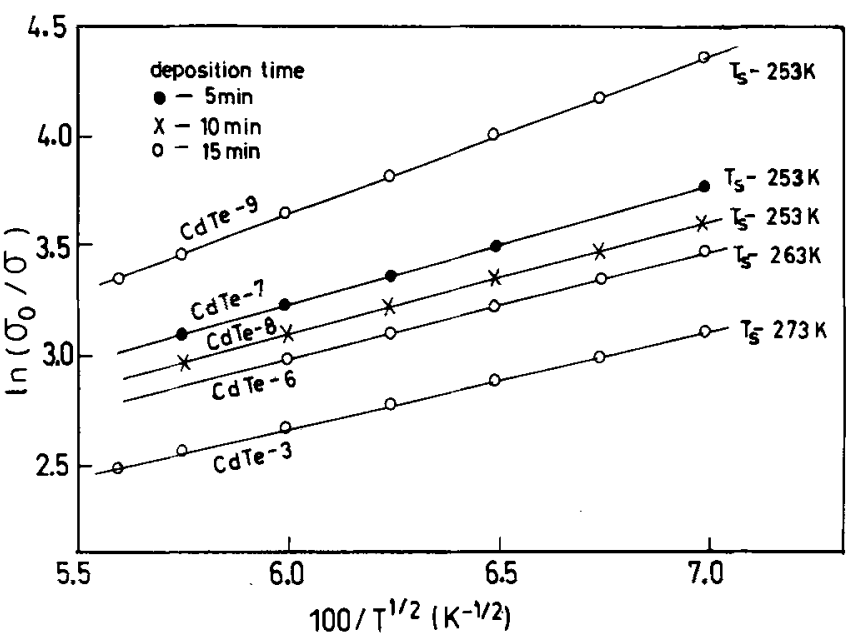

Figure 10. Plot of $\ln \left(\sigma_{0} / \sigma\right)$ vs $1 / T^{0.5}$ for CdTe films, deposited at substrate temperatures $\left(T_{\mathrm{s}}\right) \sim 253 \mathrm{~K}, 263 \mathrm{~K}$ and $\sim 273 \mathrm{~K}$ with

\begin{tabular}{|c|c|c|c|c|c|}
\hline $\begin{array}{l}\text { Sample } \\
\text { No. }\end{array}$ & $\begin{array}{c}T_{\mathrm{s}} \\
(\mathrm{K})\end{array}$ & $\begin{array}{c}T_{0} \\
(\mathrm{~K})\end{array}$ & $\begin{array}{l}W_{\mathrm{opt}} \\
(\mathrm{eV})\end{array}$ & $\begin{array}{c}R_{\mathrm{opt}} \\
(\mathrm{nm})\end{array}$ & $\underset{(\mathrm{eV})}{\Delta}$ \\
\hline \multicolumn{6}{|c|}{ Deposition time $=5 \mathrm{~min}$} \\
\hline $\mathrm{CdTe}-1$ & 273 & 1638 & 0.0288 & 4.082 & 0.0276 \\
\hline $\mathrm{CdTe}-4$ & 263 & 2673 & 0.0361 & 3.250 & 0.0352 \\
\hline $\mathrm{CdTe}-7$ & 253 & 2889 & 0.0368 & $3 \cdot 188$ & 0.0360 \\
\hline \multicolumn{6}{|c|}{ Deposition time $=10 \mathrm{~min}$} \\
\hline CdTe-2 & 273 & 1056 & 0.0230 & 5.079 & 0.0221 \\
\hline CdTe-5 & 263 & 1806 & 0.0297 & 3.956 & 0.0289 \\
\hline $\mathrm{CdTe}-8$ & 253 & 2642 & 0.0352 & 3.338 & 0.0350 \\
\hline \multicolumn{6}{|c|}{ Deposition time $=15 \mathrm{~min}$} \\
\hline $\mathrm{CdTe}-3$ & 273 & 1980 & 0.0317 & 3.707 & 0.0303 \\
\hline $\mathrm{CdTe}-6$ & 263 & 2460 & 0.0346 & 3.389 & 0.0338 \\
\hline CdTe-9 & 253 & 3685 & 0.0416 & 2.820 & 0.0413 \\
\hline
\end{tabular}
deposition times of 5,10 and $15 \mathrm{~min}$.

Table 1. Values of different parameters obtained by using ES model for CdTe films deposited at different substrate temperatures $\left(T_{\mathrm{s}}\right)$.

When $W_{\text {opt }}$ becomes comparable or greater than the Coulomb gap $(\Delta)$, there will be transition from ES hopping to Mott's hopping and the electrons will use the background or flat density of states. The optimum hopping distance $\left(R_{\mathrm{opt}}\right)$, the mean tunnelling exponent $\left(\alpha_{\mathrm{m}}\right)$, and the optimum hopping energy $\left(W_{\mathrm{op}}\right)$ were estimated from (14), (15) and (20) in the nanocrystalline CdTe films having different grain sizes. The width of the Coulomb gap $(\Delta)$ was obtained (table 1) from the relation (Ionov et al 1983; Aleshin et al 1985):

$$
\Delta=k / 2\left(T_{0} T^{*}\right)^{1 / 2},
$$

where $T^{*}$ is the temperature at which (11) begins to be satisfied. In our films we have taken $T^{*}=260 \mathrm{~K}$ since 
at this temperature there is a kink in the $\ln \sigma$ vs $1 / T$ plot corresponding to the changes in the slopes (figure 8). Now $T^{*}$ is related to $g_{0}$, the Mott's density of localized states at the Fermi level (which remains unperturbed by the Coulomb interaction), through the following equation:

$$
g_{0}=\left(k^{2} \varepsilon_{\mathrm{r}}^{3}\right) / 16 e^{6}\left(T_{0} T^{*}\right)
$$

By using the values of $T_{0}$, we have computed the $g_{0}$ values for the CdTe films which are shown in figure 11 . Lowering of defect density in these films could be observed with both decrease in substrate temperature $\left(T_{s}\right)$ or an increase of deposition time (or film thickness).

During the deposition of the nanocrystalline $\mathrm{CdTe}$ layer, the initial growth is constrained by the substrate/film interface and this resulted in a large number of defect states which were accommodated in the films. So, lower thickness films had higher defect states or $g_{0}$ values. But, with the increase of thickness the defect states were reduced due to the fact that the adatoms arriving at the substrate (to form the second layer) see the initial CdTe layer instead of the bare substrate surface. This results in a decrease of grain size $(d)$ with the increase of deposition time $(t)$, as shown in figure $7 \mathrm{a}$. This facilitates the formation of nanostructured $\mathrm{CdTe}$ layer with the desired thickness having low crystallite size as well as lower defect content.

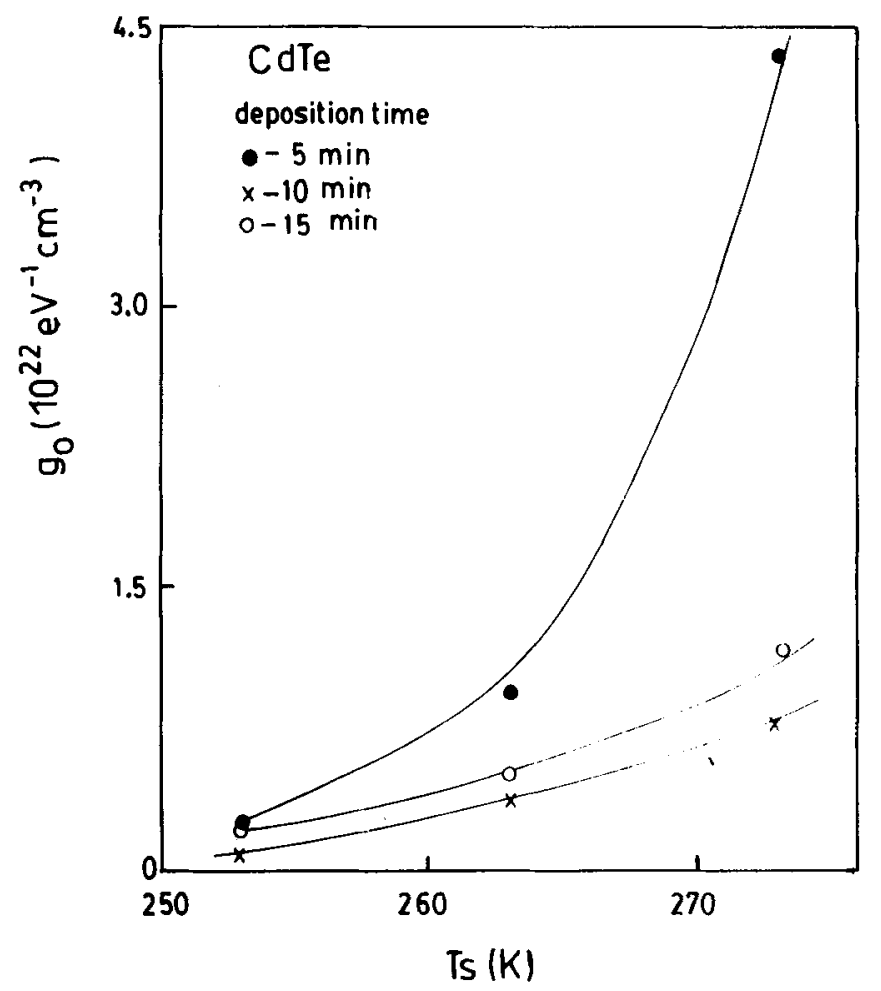

Figure 11. Variation of defect density $\left(g_{0}\right)$ with deposition temperature $\left(T_{\mathrm{s}}\right)$ for nano CdTe films with deposition times of 5,10 and $15 \mathrm{~min}$.
It would be interesting to see the applicability of Mott's $T^{025}$ relation which is widely accepted after the works of Ambagaokar et al (1971) and Pollak (1972). Figure 12 shows the plots of $\ln \sigma$ vs $1 / T^{0.25}$ for several CdTe films, the slopes of which would give $\beta \alpha_{\mathrm{m}}^{3} / \mathrm{kg}_{0}$. Using $\beta=16$ (Mott and Davis 1979) and the value of $g_{0}$ from figure 11 , we have estimated the decay length $\alpha_{\mathrm{m}}^{-1}$. It should be mentioned here that there is considerable discrepancy as regards the value of the coefficient $\beta$. The different values of $\beta$ reported so far by different workers are $16,1.5$ and $24 / \pi \quad(=7.6)$ (Mott and Davis 1979; Mott 1990, 1993), $18 \cdot 1$ (Castner 1990), $21 \cdot 1$ (Skal and Shklovskii 1974) and 27 (Ortuno and Pollak 1983).

We have studied the variation of decay length $\left(\alpha_{\mathrm{m}}^{-1}\right)$ with $T_{\mathrm{s}}$ for different thicknesses (or deposition times) of CdTe films. The decay length $\left(\alpha_{\mathrm{m}}^{-1}\right)$ decreased with lowering of $T_{\mathrm{s}}$ due to the decrease of the short range order at lower substrate temperature. This is due to the decrease of the defect density or $g_{0}$ value at lower $T_{\mathrm{s}}$ which resulted in an increase of the long range order. Correspondingly, there was a decrease of hopping distance $\left(R_{\mathrm{opp}}\right)$ and increase of hopping energy $\left(W_{\mathrm{opt}}\right)$ at lower substrate temperature and higher thicknesses which is evident from table 1.

Now, for the hopping model to be applicable in nanocrystalline CdTe films, the following criteria are to be satisfied:

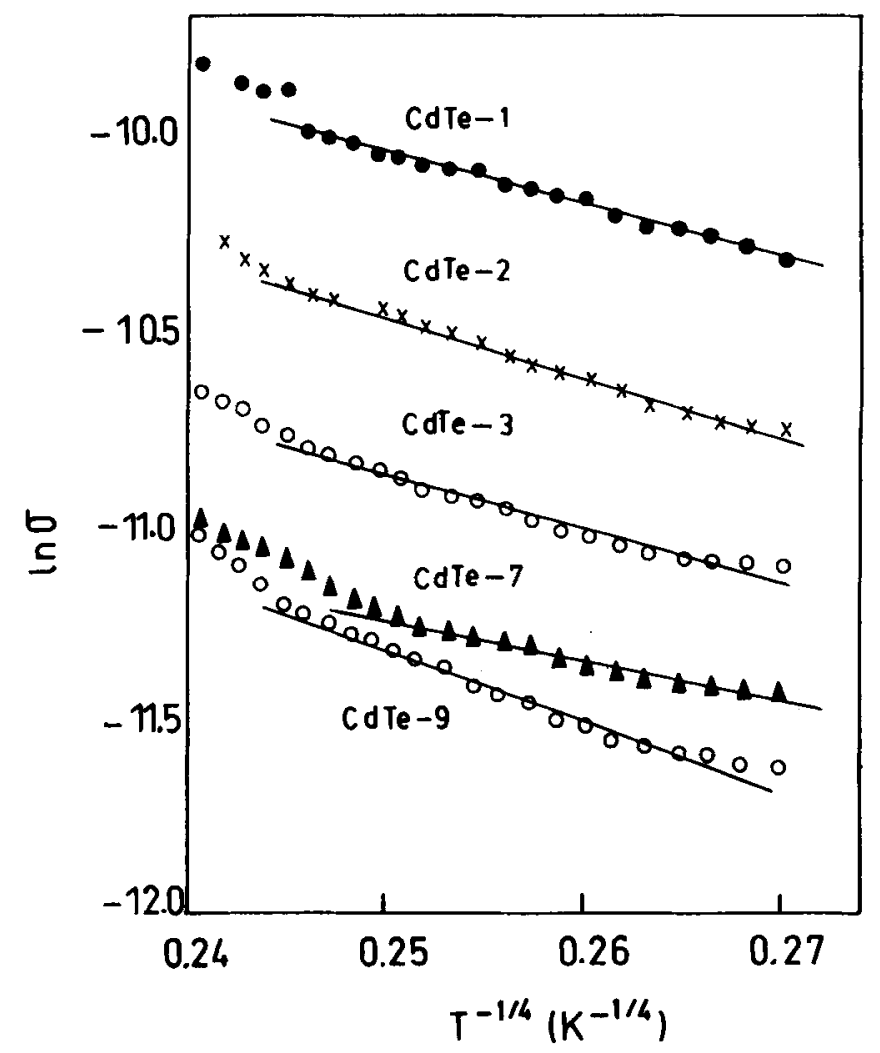

Figure 12. Variation of In $\sigma$ with $1 / T^{025}$ for nanocrystalline CdTe films. 

(i) $W_{\text {opt }} \geq k T$ or $T_{0} \geq T$,
(ii) $R_{\text {opt }} \geq d$
(iii) $\Delta>W_{\mathrm{opt}}, \Delta \gg k T$.

It appears from the values of the different parameters evaluated for our films that the first criterion in (23) is satisfied but the optimum hopping distance $R_{\mathrm{opt}}$ is comparable to the average grain size $(d)$ indicating that the electron wave function does not spread over a reasonable number of grains. As $R_{\text {opt }}$ approaches the value of $d$, the hopping process would correspond to near neighbour tunnelling and this would give rise to simple activation of carriers. In our films, the Coulomb gap $(\Delta) \sim W_{\text {opt }}$ although $\Delta>k T$ and $T_{0}>T$. So, the condition (iii) is only partially satisfied. Now, in such a situation, processes corresponding to the constant density of states outside the Coulomb gap becomes dominant and one would expect Mott's relation (13) to be valid. We have observed that in our films Mott's hopping could also explain the experimental data. In principle, one would expect both Mott and ES variable range hopping (VRH) operative on the same sample in different ranges of temperature. The cross-over temperature between the two types of VRH is determined by the half width of the Coulomb gap. But, in practice, it often becomes very difficult to distinguish between the Mott's hopping and EfrosShklovskii hopping unless the data is recorded with small variation of temperature on a wider temperature scale. The attention of the researchers was attracted in this direction after the introduction of the theoretical cross-over formalism by Aharony et al (1992). Recently, Rosenbaum et al (1996) have considered the Mott-EfrosShklovskii resistivity cross-over for three-dimensional system. Although the Mott-ES cross-over was reported for several materials (Zhang et al 1990; Rosenbaum 1991), no such studies are available for low dimensional CdTe films.

\section{Conclusions}

The sequential sputtering technique was found to be very useful in obtaining nanocrystalline films with preassigned thickness and controlled grain size throughout the thickness $(\sim 40 \mathrm{~nm}$ or less). Nanocrystalline $\mathrm{ZnS}$ films exhibited zinc blende structure and films with different crystallite sizes could be obtained. Thicknesses of the films could also be varied without sacrificing the blue shift. The photoluminescence (PL) studies of nano $\mathrm{ZnS}$ films suggested that transitions from energy states in the band gap were favoured for luminescence process which could be attributed to the surface states present in the films.

Electrical conductivities of CdTe films deposited on quartz substrates indicated a $\left(T_{0} / T\right)^{p}$ variation. The average value of the exponent $p$ was $\sim 0.5$ which indicated variable range hopping within the Coulomb gap (EfrosShklovskii hopping) to be the dominant process of conduction in the nanocrystalline CdTe films. The width of the Coulomb gap was within $0.02-0.04 \mathrm{eV}$ which depended on the deposition condition. The existing theoretical models were used for the estimation of hopping energy (0.02-0.04 eV) and hopping distance $(2.8-5.1 \mathrm{~nm})$ in the films. In CdTe films, the Coulomb gap $(\Delta) \sim W_{\text {opt }}$ although $\Delta>k T$ and $T_{0}>T$. Now, in such a situation, processes corresponding to the constant density of states outside the Coulomb gap become dominant. We have observed that in our films Mott's hopping could also explain the experimental data. The cross-over temperature between the two types of VRH is determined by the half width of the Coulomb gap.

\section{Acknowledgements}

The author wishes to acknowledge with thanks the financial support from the Council of Scientific and Industrial Research and the Department of Science and Technology, New Delhi for executing the programme.

\section{References}

Aharony A, Zhang Y, Dai P, Levy M and Sarachik M P 1992 Phys. Rev. Lett. 683900

Aleshin A N, Dvurechenskii A V, Ionov A N, Ryazantsev I A and Shlimak I S 1985 Sov. Phys. Semicond. 19759

Allegre J, Arnaud G, Mathieu H, Lefebvre P, Granier W and Boudes L 1994 J. Cryst. Growth 138998

Ambegaokar V, Halperin B I and Langer J S 1971 Phys. Rev. B4 2612

Banerjee S, Pal R, Maity A B, Chaudhuri S and Pal A K 1997 Nanostruct. Mater. 8301

Bhattacharyya D, Bhattacharyya S K, Chaudhuri S and Pal A K 1992a Vacuum 43 313, 1201

Born M and Wolf E 1959 Principles of optics (London: Pergamon Press)

Castner. T G 1990 Hopping transport in solids (eds) M Pollak and B I Shklovskii (Amsterdam: Elsevier/North Holland) p. 1

Chen W, Wang Z, Lin Z and Lin L 1997 J. Appl. Phys. 82 3111

Chestnoy N, Harris T D, Hull R and Brus L E 1986 J. Phys. Chem. 90339

Demichelis F, Pirri C F and Tresso E 1993 Philos. Mag. B67 331

Efros A L and Shklovskii B I 1975 J. Phys. C8 L49

Graham M R, Bellingham J R and Adkins C J 1992 Philos. Mag. B65 669

Gupta P, Pal R, Bhattacharyya D, Chaudhuri S and Pal A K 1995 Phys. Status Solidi (a)148 459

Ionov A N, Shlimak I S and Matveev M N 1983 Solid State Commun. 47763

Kayanuma Y 1988 Phys. Rev. B38 9797 
Klein M C, Hache F, Ricard D and Flytzanis C 1990 Phys. Rev. B42 11123

Maity A B, Bhattacharyya D, Sharma S R, Chaudhuri S and Pal A K 1995 Nanostruct. Mater. 5717

Mandal S K, Chaudhuri S and Pal A K 1998 Nanostruct. Mater. 10607

Mott N F 1968 J. Non-Cryst. Solids 1 I

Mott N F 1990 Metal-insulator transitions (London: Taylor and Francis) p. 50

Mott N F 1993 Conduction in non-crystalline materials (Oxford: Clarendon)

Mott N F and Davis E A 1979 Electronic processes in noncrystalline materials (Oxford: Clarendon Press) p. 221

Ortuno M and Pollak M 1983 J. Non-Cryst. Solids 59 \& 6053

Pal R, Bhattacharyya D, Maity A B, Chaudhuri S and Pal A K 1994 Nanostruct. Mater. 4329

Pollak M 1972 J. Non-Cryst. Solids 8-10 486

Rosenbaum R 1991 Phys. Rev. B44 3599

Rosenbaum R, Lien N V, Grahnam M R and Witcomb M 1996 J. Phys. Condens. Matter 96247
Schmitt-Rink S, Miller D A B and Chemla D S 1987 Phys. Rev. B35 8113

Schmitt-Rink S, Chemla D S and Miller D A B 1989 Adv. Phys. 3889

Sekikawa T, Yao H, Hayashi T and Kobayashi T 1992 Solid State Commun. 83969

Shklovskii B I and Efros A L 1984 Electronic properties of doped semiconductors (Berlin: Springer)

Skal A S and Shklovskii B I 1974 Sov. Phys. Solid State 16 1190

Tsang W T 1987 Semiconductors and semimetals (eds) R K Willardson and A C Beer (New York: Academic Press) vol. 24 p. 397

Woggon U and Gaponenko S V 1995 Phys. Status Solidi B189 285

Yoffe A D 1993 Adv. Phys. 42173

Zabrodski A G and Zinoveva K N 1984 Sov. Phys. JETP 59 425

Zhang Y, Dai P, Levy M and Sarachik M P 1990 Phys. Rev. Lett. 642688 